\section{BBB-conquering antibodies}

\section{By Benjamin Boettner, Associate Editor}

Roche has developed a new transferrin receptor-targeting strategy that dramatically increased delivery of an antibody to the brain. ${ }^{1}$ The key advance was slimming the technology, dubbed Brain Shuttle, down to a single transferrin receptor-binding antibody fragment. The pharma showed proof of concept in mice by delivering an anti- $\beta$-amyloid $\mathrm{mAb}$ and now needs to adapt the system for primate brains.

The blood brain barrier (BBB) is composed of endothelial cells that allow the passive exchange of smaller, lipophilic molecules between blood and the brain parenchyma but are impermeable to larger, more hydrophobic molecules such as many biologics.

BBB endothelial cells have evolved dedicated, receptor-mediated transcytosis pathways to shuttle larger endogenous molecules such as transferrin and insulin. ${ }^{2}$ Because transferrin is one of the few macromolecules able to trek across the BBB, researchers have developed Trojan horse delivery systems that harness transferrin receptor (TfR)mediated transcytosis to shuttle biologics into the brain.

First-generation Trojan horses typically consisted of an entire TfRbinding antibody fused to a payload-usually a second antibody against a disease-related target. However, the high affinity of the antibody for TfR led to insufficient target engagement in the brains of animals. ${ }^{3}$

In 2011, Roche's Genentech Inc. unit took steps toward addressing both drawbacks by lowering the affinity of a TfR-directed antibody. The result was improved brain uptake.

Genentech then eliminated the need to fuse two antibodies together by developing a bispecific, chimeric antibody. One antigen-binding site was specific for TfR and the other for $\beta$-site APP-cleaving enzyme 1 (BACE1), an enzyme that contributes to processing the amyloid precursor protein (APP) into toxic, amyloidogenic forms.

The bispecific antibody achieved brain concentrations of about $20 \mathrm{nM}$, which was up to 10 -fold more than a monospecific, BACE1directed antibody, and reduced $\beta$-amyloid $(A \beta)$ levels in a mouse model of Alzheimer's disease (AD). ${ }^{4}$

Now, the Roche team, led by Per-Ola Freskgård and Anirvan Ghosh, has taken a different approach. The group took a therapeutic antibody called gantenerumab and fused a TfR-specific antibody fragment to one (monovalent) or both (bivalent) of gantenerumab's heavy chains.

Freskgård is vice director and senior leader for neuroscience at the Roche Pharma Research and Early Development (pRED) section, and Ghosh is head of neuroscience discovery at pRED. Gantenerumab, a HuCAL (Human Combinatorial Antibody Library)-derived mAb targeting $\mathrm{A} \beta$, is partnered with MorphoSys AG and is in Phase II/III testing to treat $\mathrm{AD}$.

The monovalent version produced the better results-it was efficiently transcytosed via TfRs across the BBB.

In a mouse model of $\mathrm{AD}$, i.v. injections of the monovalent, TfRbinding antibody resulted in 50-fold increased target engagement in amyloid plaques in the brain compared with gantenerumab. The monovalent antibody markedly reduced plaque burden in the animals' cortex and hippocampal brain regions.

The findings were published in Neuron.

"The most important finding is that endothelial cells at the BBB differentially process internalized TfR in response to monovalent versus bivalent binding of the receptor. Monovalent binding was preferentially associated with transcytosis, while bivalent binding preferentially led to lysosomal compartmentalization," said David Hilbert, CSO of cancer antibody company Zyngenia Inc. "This will have important consequences for the further development of brain shuttles."

Richard Daneman, a fellow at the University of California, San Francisco, added, "One of the nice things in the new monovalent configuration is that it can be used easily with other therapeutic antibodies. It represents more of a platform than other Trojan horse antibodies."

The reason, he said, is that the new Brain Shuttle technology incorporates therapeutic antibodies "with their native architecture."

"In principle, such an efficient brain shuttle can revolutionize the development of medication for neurological disorders," said Bart De Strooper, a professor at the Catholic University Leuven and head of the Department of Molecular and Developmental Genetics at the Flanders Institute for Biotechnology (VIB).

\section{Shuttle service}

Roche is already testing its Brain Shuttle technology in combination with at least two therapeutic modalities-antisense and antibodies.

Under a 2013 deal with Isis Pharmaceuticals Inc., the partners are combining Brain Shuttle with antisense molecules against huntingtin (HTT) protein to treat Huntington's disease (HD).

Also last year, Roche partnered with Prothena Corp. plc to develop a Brain Shuttle version of a mAb from the biotech to treat Parkinson's disease (PD).

Regardless of modality, De Strooper wanted to see more in vivo toxicity testing of Brain Shuttle in chronic conditions.

"TfR-specific antibodies can cause severe problems with red blood cells," he noted.

For example, anti-TfR antibodies are associated with defects in reticulocytes, immature red blood cells that express high levels of the receptor required to take up transferrin-bound iron needed for hemoglobin loading and cell maturation. ${ }^{5}$

Earlier this week, the Genentech team published data showing how high-affinity, TfR-directed antibodies such as its TfR-BACE1 bispecific, chimeric antibody drive TfR into the lysosome for degradation. Over time, TfR levels become depleted, rendering the pathway unavailable for transcytosis of endogenous molecules and biologics. ${ }^{6}$ 


\section{ANALYSIS}

In contrast, Roche's monovalent TfR format could avoid toxicity because it preserved normal TfR levels on the luminal side of the BBB and did not induce TfR degradation in mice.

Michael Ehlers, SVP and CSO of the neuroscience research unit at Pfizer Inc., wanted to see more characterization of Brain Shuttle. "So far the distribution of the antibody has only been assessed by immunofluorescence-based signal amplification," he said. "It will be important to assess the antibody fraction that reaches the brain parenchyma more directly at higher resolution in time course experiments and to investigate where and how long it is detectable."

Hilbert thinks that affinity and valency are "two sides of the same coin" and that further work will help to define the role of each

\section{"In principle, such an efficient brain shuttle can revolutionize the development of medication for neurological disorders." \\ -Bart De Strooper, \\ Catholic University Leuven} in transcytosis. It would be interesting to test Brain Shuttle and Genentech's approach head to head. "True affinities of the Trojan horse antibodies by Genentech and Roche still need to be measured by more sensitive means such as surface plasmon resonance," he said.

"It would make sense and be straightforward to compare the different TfR-based Trojan horse shuttles side by side and quantify their effective brain concentrations. In vivo assays could be used to further translate such results," added Ehlers.

Moreover, he said that "more experiments should be done in wildtype animals since mouse models of $\mathrm{AD}$ often have impaired vascular integrity." Indeed, $A \beta$ forms aggregates in the cerebrovasculature that can locally disrupt the BBB.

\section{Deciphering the human BBB}

Freskgård said that Roche is now pushing ahead with plans to generate Brain Shuttle modules with primate and human TfRs.

Daneman said that primate- or human-specific anti-TfR antibodies will likely have varying binding affinities and TfR functions.
"The BBB and the functions of individual transport systems like TfRs likely differ between rodents and primates so that efficacy seen in rodent models cannot necessarily be anticipated in humans. Therefore, a more detailed understanding about the BBB in primates will be very useful," said Ehlers.

He said that a team at the University of Wisconsin-Madison is developing human induced pluripotent stem (iPS) cell protocols that generate in vitro systems able to recapitulate aspects of the human BBB. ${ }^{7}$

Roche's $p R E D$ has filed a patent application covering BBB transport using the monovalent Brain Shuttle module. The IP is not available for licensing.

Boettner, B. SciBX 7(4); doi:10.1038/scibx.2014.104

Published online Jan. 30, 2014

\section{REFERENCES}

1. Niewoehner, J. et al. Neuron; published online Jan. 8, 2014; doi:10.1016/j.neuron.2013.10.061

Contact: Per-Ola Freskgård, Roche, Basel, Switzerland e-mail: per-ola.freskgard@roche.com

Contact: Anirvan Ghosh, same affiliation as above e-mail: anirvan.ghosh@roche.com

2. Pardridge, W.M. J. Cereb. Blood Flow Metab. 32, 1959-1972 (2012)

3. Yu, Y.J. \& Watts, R.J. Neurotherapeutics 10, 459-472 (2013)

4. Yu, Y.J. et al. Sci. Transl. Med. 3, 84ra44 (2011)

5. Couch, J.A. et al. Sci. Transl. Med. 5, 183 ra57 (2013)

6. Bien-Ly, N. et al. J. Exp. Med.; published online Jan. 27, 2014; doi:10.1084/jem.20131660

7. Lippmann, E.S. et al. Nat. Biotechnol. 30, 783-791 (2012)

COMPANIES AND INSTITUTIONS MENTIONED

Catholic University Leuven, Leuven, Belgium

Flanders Institute for Biotechnology, Leuven, Belgium

Genentech Inc., South San Francisco, Calif.

Isis Pharmaceuticals Inc. (NASDAQ:ISIS), Carlsbad, Calif. MorphoSys AG (Xetra:MOR; Pink:MPSYF), Martinsried, Germany Pfizer Inc. (NYSE:PFE), New York, N.Y.

Prothena Corp. plc (NASDAQ:PRTA), Dublin, Ireland

Roche (SIX:ROG; OTCQX:RHHBY), Basel, Switzerland

University of California, San Francisco, Calif.

University of Wisconsin-Madison, Madison, Wis.

Zyngenia Inc., Gaithersburg, Md. 